\title{
SCRLH-TL Based Sequential Rotation Feed Network for Broadband Circularly Polarized Antenna Array
}

\author{
Bin-Feng ZONG ${ }^{1}$, Guang-Ming $W A N G^{1}$, Hui-Yong ZENG ${ }^{1,2}$, Ya-Wei $W A N G^{1}$, Ding WANG ${ }^{3}$ \\ ${ }^{1}$ Air and Missile Defense College, Air Force Engineering University, Shaanxi 710051, China \\ ${ }^{2}$ Unit 93942, Shaanxi 712000, China \\ ${ }^{3}$ Qingdao Campus of Naval Aero Engineering Academy, Shandong 266041, China
}

zongbinfeng@sina.com,wgming01@sina.com

Manuscript received June 29, 2015

\begin{abstract}
In this paper, a broadband circularly polarized (CP) microstrip antenna array using simplified composite right/left-handed transmission lines (SCRLH-TLs) based sequential rotation (SR) feed network is presented. The characteristics of a SCRLH-TL are investigated initially. Then, a broadband low insertion loss $45^{\circ}$ phase shifter is designed using the SCRLH-TL and the phase shifter is employed in constructing a SR feed network for CP antenna array. To validate the design method of the SR feed network, a $2 \times 2$ antenna array comprising sequentially rotated coupled stacked CP antenna elements is designed, fabricated and measured. Both the simulated and measured results indicate that the performances of the antenna element are further enhanced when the SR network is used. The antenna array exhibits the VSWR less than 1.8 from $4 \mathrm{GHz}$ to $7 \mathrm{GHz}$ and the axial ratio (AR) less than $3 \mathrm{~dB}$ from $4.4 \mathrm{GHz}$ to $6.8 \mathrm{GHz}$. Also, high peak gain of $13.7 \mathrm{dBic}$ is obtained. Besides, the normalized radiation patterns at the operating frequencies are symmetrical and the sidelobe levels are low at $\varphi=0^{\circ}$ and $\varphi=90^{\circ}$.
\end{abstract}

\section{Keywords}

Simplified composite right/left-handed transmission line (SCRLH-TL), circularly polarized (CP), broadband, sequential rotation (SR) feed network

\section{Introduction}

Due to their low profile, easy fabrication and flexibility in orientation angle between transmitter and receiver antennas, circularly polarized (CP) microstrip antenna arrays have become an excellent choice for applications in communication systems such as satellite, mobile, radar tracking and the remote control and telemetry [1-3]. However, many disadvantages, such as narrow bandwidth and relatively high-fed line losses, are often along with the merits. Recently, many techniques have been investigated to overcome these disadvantages. Among them, sequential rotation (SR) feed technique has been proved an effective way to design $\mathrm{CP}$ antenna arrays with broadband, high polarization purity and symmetric radiation patterns. When the SR techniques are used, the radiated polarization type of the element cannot be considered and the overall bandwidth performances of $\mathrm{CP}$ antenna arrays can be significantly improved. Until now, many CP antenna arrays using SR feed networks have been reported [4-10]. In [4], CP antenna array with wide axial ratio (AR) bandwidth was obtained by rotating linearly polarized elements sequentially. However, the use of linearly polarized elements leads to a high gain loss. In [5], two simple and compact sequential-phase feed networks for CP SR arrays were presented. The proposed SR feed networks could extend the bandwidth of the AR and improve the gain-level obviously. However, the narrow bandwidth limits its application in wideband systems. In [6], a novel broadband CP antenna array was presented using SR feed technique. Instead of using linearly polarized elements in [4], [7] and [8], CP antenna elements were used to improve the AR bandwidth. However, the defected ground structure leads to back radiation and difficult encapsulation. In [9], a Ka-band CP microstrip antenna array with a high-gain level was designed. The serial feed SR technique improved the bandwidth, radiation pattern, and polarization purity over a wider frequency band compared with the coplanar corporate feed network. In [10], three different types of wideband feeding networks, including parallel feeding network, series feeding network and hybrid ring feeding network, were studied to optimize the CP bandwidth. For the CP dielectric resonator antenna subarray with hybrid ring feeding network, the impedance matching bandwidth $\left(\left|\mathrm{S}_{11}\right|<10 \mathrm{~dB}\right)$ and 3-dB AR bandwidth achieved were $44 \%$ and $26 \%$, respectively.

In this paper, a novel method to design SR feed network for a broadband CP microstrip antenna array is presented. The network employs a balun and two orthogonal power dividers. The balun is achieved by back-connecting a conventional in-phase power divider and a $180^{\circ}$ phase shifter together, and the orthogonal power divider is achieved by back-connecting a conventional in-phase power divider and a $90^{\circ}$ phase shifter together. Both the $180^{\circ}$ phase shifter and the $90^{\circ}$ phase shifter are designed using the reported simplified composite right/left-handed transmission lines (SCRLH-TLs) [11], [12]. To validate the design methodology, four coupled stacked CP antenna ele- 
ments are excited rotationally by the designed network to form a CP antenna array. The experiment results show that the antenna array based on the new SR feed network obtained not only a wide AR bandwidth, but also a high gain level. Besides, the easy fabrication of the antenna array makes it very useful in broadband systems.

\section{Broadband SR Feed Network Based on SCRLH-TL}

\subsection{Phase Shifter using SCRLH-TL}

As reported in [11], a SCRLH-TL, which has a nonlinear phase response, was proposed. The distributed microstrip structure and its corresponding equivalent lumped circuit model are shown in Fig. 1(a) and Fig. 1(b), respectively. Referring to the equivalent lumped circuit model, the shunt capacitance $C_{\mathrm{R}}$ and a part of series inductance $L_{\mathrm{R}}$ are provided by a wide microstrip line, while the other part of $L_{\mathrm{R}}$ is realized by a short narrow microstrip line. The shunt inductance $L_{\mathrm{L}}$ is implemented by a narrow short stub.

Based on the Bloch-Floquet theory, the dispersion relation can be expressed as

$$
\beta p=\cos ^{-1}\left(1+\frac{Z(\omega) \cdot Y(\omega)}{2}\right)
$$

where $\beta$ is the propagation constant, $p$ is the physical length of the SCRLH-TL, $\omega$ is the working frequency, and $Z(\omega)$ and $Y(\omega)$ are the series impedance and the shunt admittance of the SCRLH-TL, respectively.

$$
\begin{gathered}
Z(\omega)=j \omega\left(\frac{L_{R}^{2}}{4 L_{L}}+L_{R}\right) \\
Y(\omega)=j \omega C_{R}+\frac{2}{j \omega\left(\frac{L_{R}}{2}+2 L_{L}\right)}
\end{gathered}
$$

Combining (1)-(3), when $\beta p=0$,

$$
\omega=\omega_{1}=\frac{1}{\sqrt{\left(L_{L}+\frac{L_{R}}{4}\right) C_{R}}}
$$

And when $\beta p=\pi$,

$$
\omega=\omega_{2}=\omega_{1} \sqrt{1+\frac{4 L_{L}}{L_{R}}} .
$$

So, the transmission bandwidth of the SCRLH-TL is determined by $\omega_{1}$ and $\omega_{2}$.

If the operating band of the TL is from $\omega_{1}$ to $\omega_{2}$, the differential phase shift $\Delta \Phi$ of the output signal compared to a normal microstrip TL of electrical length $\theta_{r}$ can be expressed as

$$
\Delta \Phi=\theta_{r}-\cos ^{-1}\left(1+\frac{Z(\omega) \cdot Y(\omega)}{2}\right) .
$$

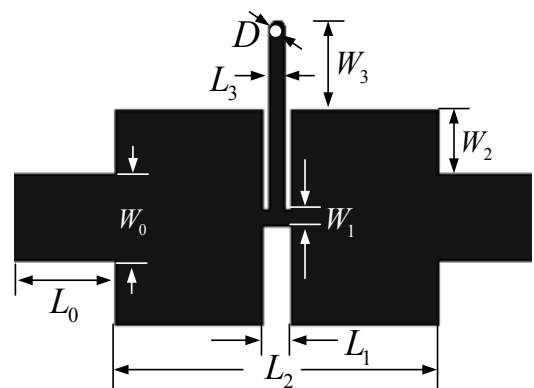

(a)

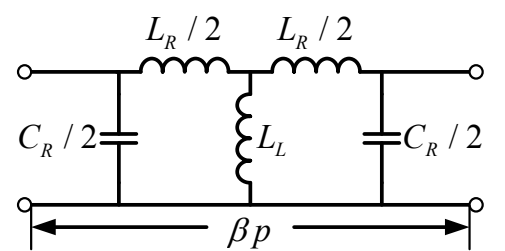

(b)

Fig. 1. SCRLH-TL without a series capacitor. (a) Distributed structure. (b) Equivalent lumped circuit model.

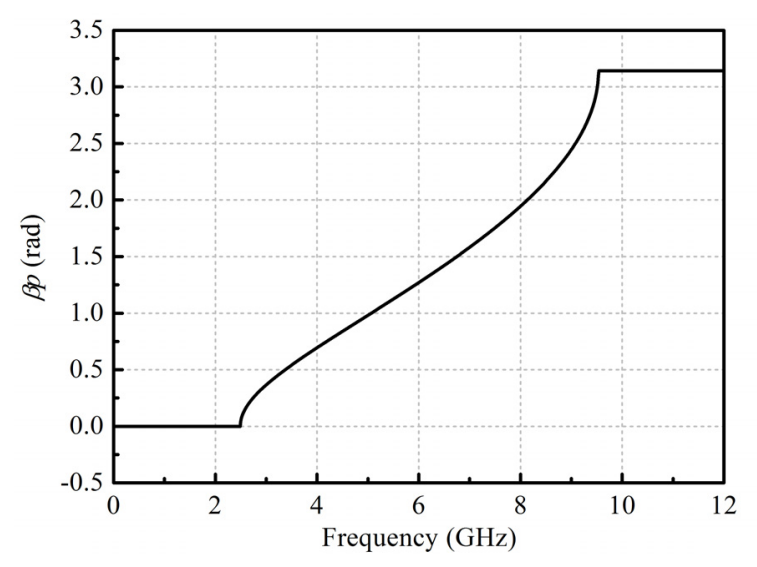

Fig. 2. Dispersion curve for the SCRLH-TL.

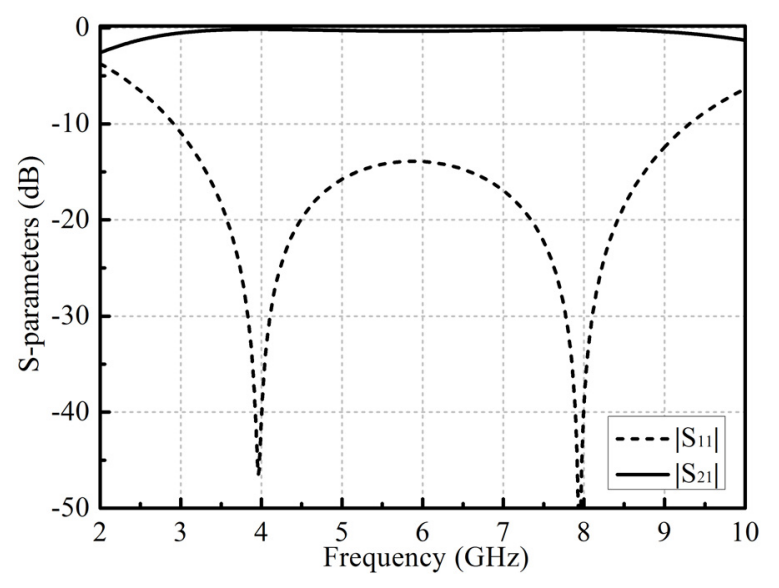

Fig. 3. S-parameters of the SCRLH-TL.

$\theta_{r}$ is defined as

$$
\theta_{r}=\theta_{r 0} \frac{f}{f_{0}} .
$$

where, $\theta_{r 0}$ is the electrical length of the reference line at frequency $f_{0}$. 


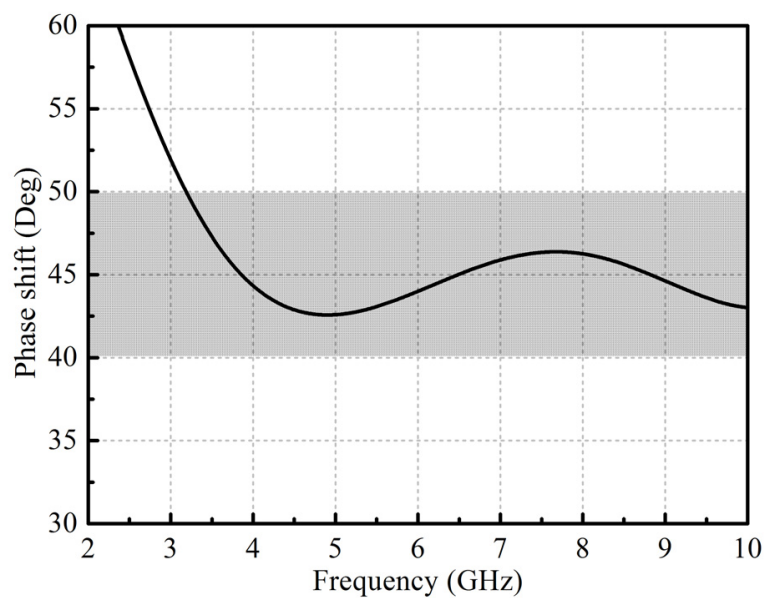

Fig. 4. Phase response of the $45^{\circ}$ phase shifter.

In this paper, the design destinations are $f_{0}=6 \mathrm{GHz}$ and $\Delta \Phi=45^{\circ}$, and the lumped elements are chosen as: $L_{R}=1.12 \mathrm{nH}, C_{R}=1 \mathrm{pF}$, and $L_{L}=3.8 \mathrm{nH}$. The dispersion curve, which is calculated using the values of the lumped elements, is shown in Fig. 2. The curve indicates that the bandwidth of the SCRLH-TL is from $2.49 \mathrm{GHz}$ to 9.51 GHz and the SCRLH-TL has a nonlinear phase response in band. According to the values of the lumped elements, the dimensions of the SCRLH-TL are optimized as: $L_{0}=3.5 \mathrm{~mm}, L_{1}=0.9 \mathrm{~mm}, L_{2}=6.5 \mathrm{~mm}, L_{3}=0.4 \mathrm{~mm}, W_{0}=$ $1.36 \mathrm{~mm}, W_{1}=0.4 \mathrm{~mm}, W_{2}=1.32 \mathrm{~mm}, W_{3}=2.45 \mathrm{~mm}$, $D=0.3 \mathrm{~mm}$. The required physical length of the $50 \Omega$ normal TL is $17.46 \mathrm{~mm}$. A substrate with a dielectric constant of 2.65 and a thickness of $0.5 \mathrm{~mm}$ is used. The simulated results of the $45^{\circ}$ phase shifter are depicted in Fig. 3 and Fig. 4. The return loss of the SCRLH-TL from $2.91 \mathrm{GHz}$ to $9.31 \mathrm{GHz}$ is better than $10 \mathrm{~dB}$, and a differen tial phase shift of $45^{\circ} \pm 5^{\circ}$ is achieved from $3.18 \mathrm{GHz}$ to $10 \mathrm{GHz}$. So, the designed phase shifter can be used for the band from $3.18 \mathrm{GHz}$ to $9.31 \mathrm{GHz}$.

\subsection{Theory of the SR Feed Network}

Figure 5 presents the sketch map of the SR feed network. $\mathrm{a}_{i}, \mathrm{~d}_{m}(i=1,2, \ldots, 5 ; m=1,2)$ are normalization incident wave voltages and $\mathrm{b}_{i}, \mathrm{c}_{m}(i=1,2, \ldots, 5 ; m=1,2)$ are normalization reflection wave voltages. It can be seen that the network consists of a balun and two orthogonal power dividers. Based on the principle of microwave network [13], the $S$-parameters of a balun and an orthogonal power divider can be expressed as

$$
\mathbf{S}_{\text {balun }}=\frac{1}{\sqrt{2}}\left[\begin{array}{ccc}
0 & -j & j \\
-j & 0 & 0 \\
j & 0 & 0
\end{array}\right], \quad \mathbf{S}_{\text {orth. }}=\frac{1}{\sqrt{2}}\left[\begin{array}{ccc}
0 & -j & 1 \\
-j & 0 & 0 \\
1 & 0 & 0
\end{array}\right] .
$$

These two networks are matched at the input ports and have transmission coefficients of $-3 \mathrm{~dB}$ at two output ports of them. The balun has an opposite phase response and the orthogonal power divider has an orthogonal phase response between their output ports. Based on these two expressions, the following relations for the SR feed network can be obtained

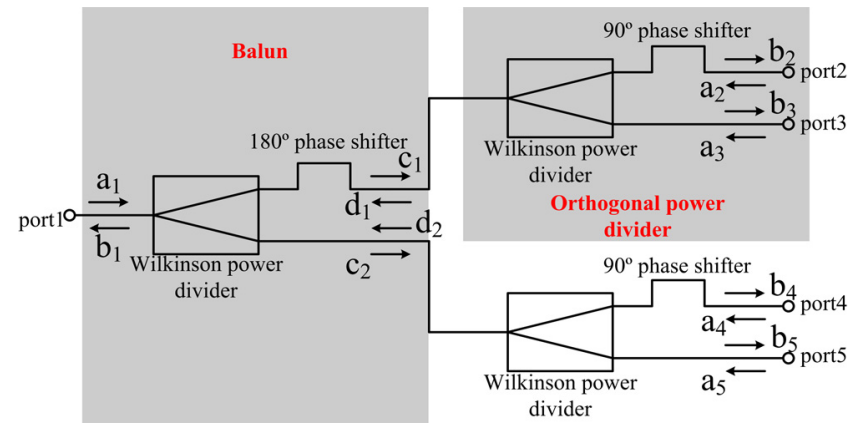

Fig. 5. The sketch map of the SR feed network.

$$
\begin{aligned}
& {\left[\begin{array}{l}
b_{1} \\
c_{1} \\
c_{2}
\end{array}\right]=\frac{1}{\sqrt{2}}\left[\begin{array}{ccc}
0 & j & -j \\
j & 0 & 0 \\
-j & 0 & 0
\end{array}\right]\left[\begin{array}{l}
a_{1} \\
d_{1} \\
d_{2}
\end{array}\right],} \\
& {\left[\begin{array}{l}
d_{1} \\
b_{1} \\
b_{3}
\end{array}\right]=\frac{1}{\sqrt{2}}\left[\begin{array}{ccc}
0 & -1 & j \\
-1 & 0 & 0 \\
j & 0 & 0
\end{array}\right]\left[\begin{array}{l}
c_{1} \\
a_{2} \\
a_{3}
\end{array}\right],} \\
& {\left[\begin{array}{l}
d_{2} \\
b_{4} \\
b_{5}
\end{array}\right]=\frac{1}{\sqrt{2}}\left[\begin{array}{ccc}
0 & -1 & j \\
-1 & 0 & 0 \\
j & 0 & 0
\end{array}\right]\left[\begin{array}{l}
c_{2} \\
a_{4} \\
a_{5}
\end{array}\right] .}
\end{aligned}
$$

Combining the expressions in (9), the relation between the normalization incident wave voltages $a_{i}$ and normalization reflection wave voltages $b_{i}$ of the SR feed network can be achieved

$$
\left[\begin{array}{l}
b_{1} \\
b_{2} \\
b_{3} \\
b_{4} \\
b_{5}
\end{array}\right]=\frac{1}{2}\left[\begin{array}{ccccc}
0 & -j & -1 & j & 1 \\
-j & 0 & 0 & 0 & 0 \\
-1 & 0 & 0 & 0 & 0 \\
j & 0 & 0 & 0 & 0 \\
1 & 0 & 0 & 0 & 0
\end{array}\right]\left[\begin{array}{l}
a_{1} \\
a_{2} \\
a_{3} \\
a_{4} \\
a_{5}
\end{array}\right] .
$$

Namely, the $S$-parameters of the SR feed network is

$$
\mathbf{S}_{\mathrm{SR} \text { network }}=\frac{1}{2}\left[\begin{array}{ccccc}
0 & -j & -1 & j & 1 \\
-j & 0 & 0 & 0 & 0 \\
-1 & 0 & 0 & 0 & 0 \\
j & 0 & 0 & 0 & 0 \\
1 & 0 & 0 & 0 & 0
\end{array}\right] \text {. }
$$

From (11), it can be seen that the SR feed network provides $90^{\circ}$ phase shift between adjacent output ports, which can be used for generating circular polarization with a wide AR bandwidth.

\subsection{Synthesizing of SR Feed Network}

Based on the theory, a broadband SR feed network, which consists of a balun and two orthogonal power dividers, is designed. The distributed model of the proposed SR feed network is presented in Fig. 6. The balun is synthesized using a Wilkinson power divider and a $180^{\circ}$ phase shifter, and the orthogonal power divider is synthesized using a Wilkinson power divider and a $90^{\circ}$ phase shifter. 


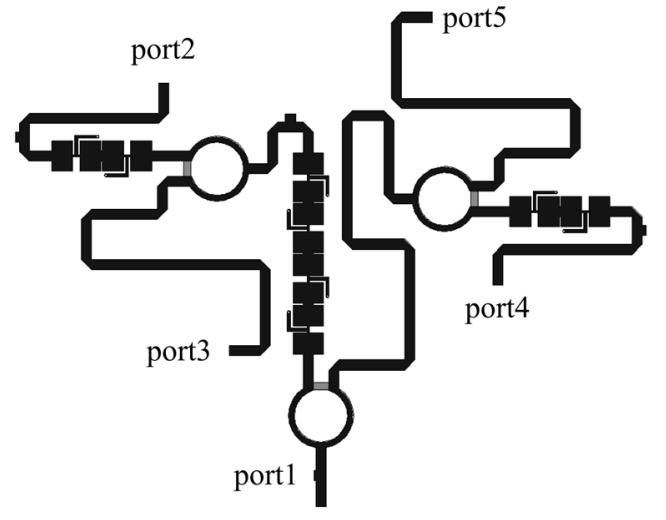

Fig. 6. Distributed model of the SR feed network using the SCRLH-TLS.

Here, two $45^{\circ}$ phase shifters are cascaded for $90^{\circ}$ phase shifter and four $45^{\circ}$ phase shifters are cascaded for $180^{\circ}$ phase shifter.

The simulated results of the SR feed network using the SCRLH-TLs are depicted in Fig. 7. For comparison, the results of a SR feed network using the normal microstrip TLs are also depicted. From Fig. 7(a) and (b), it can be seen that the SR feed network using the SCRLH-TLs achieves a differential magnitude of $7.7 \pm 0.65 \mathrm{~dB}$ from

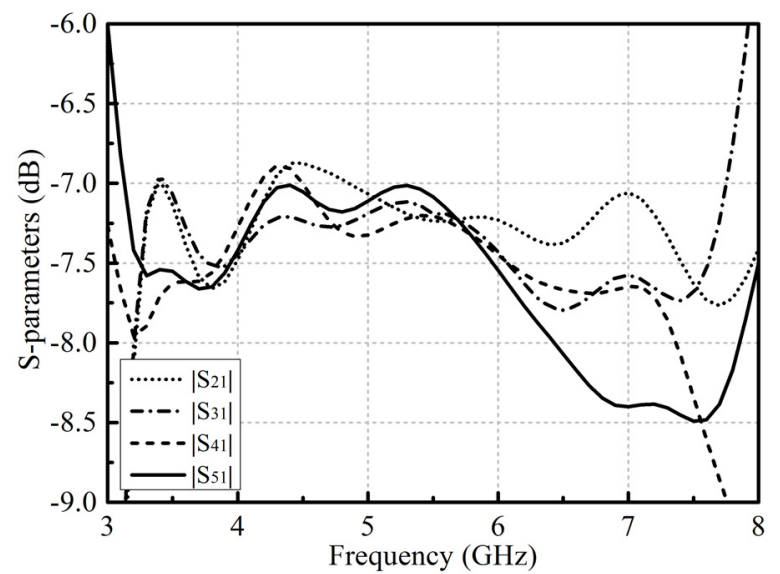

(a)

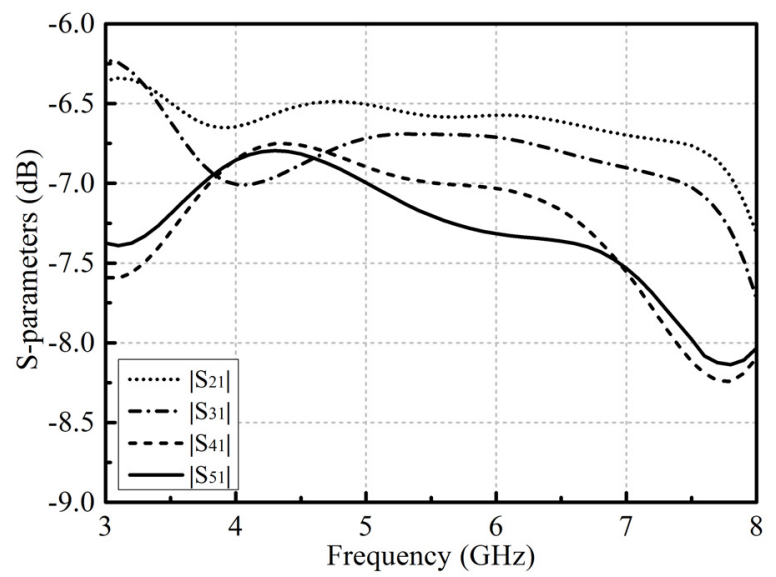

(c)
3.5 GHz to $7.5 \mathrm{GHz}$ and a differential phase shift of $90^{\circ} \pm 10^{\circ}$ from $3 \mathrm{GHz}$ to $8 \mathrm{GHz}$. And from Fig. 7(c) and (d), it can be seen that the SR feed network based on the normal TLs achieves a differential magnitude of $7.25 \pm 0.75 \mathrm{~dB}$ from $3.5 \mathrm{GHz}$ to $7.5 \mathrm{GHz}$ and a differential phase shift of $90^{\circ} \pm 10^{\circ}$ from $5 \mathrm{GHz}$ to $5.9 \mathrm{GHz}$. The results show that the SR feed network based on SCRLH-TLs has a broader band than that of the network using the normal ones.

\section{Antenna Element}

To design a broadband SR antenna array, the radiating element should satisfy the requirements including: 1) A broadband low VSWR level. Elements with a broadband low VSWR level cannot only reduce the unbalance influence of the feed network, but also improve the array radiation efficiency. 2) Good CP performance and a broad AR bandwidth. The antenna array based on radiation elements with good CP performance and a broad AR bandwidth has a better ability to distinguish the cross-polarization and reduce the loss of cross-polarization. 3) Convenient to form array and easy to be fabricated. When we design a radiation element for SR antenna array, the cost and the complexity of fabrication should be taken into conside-

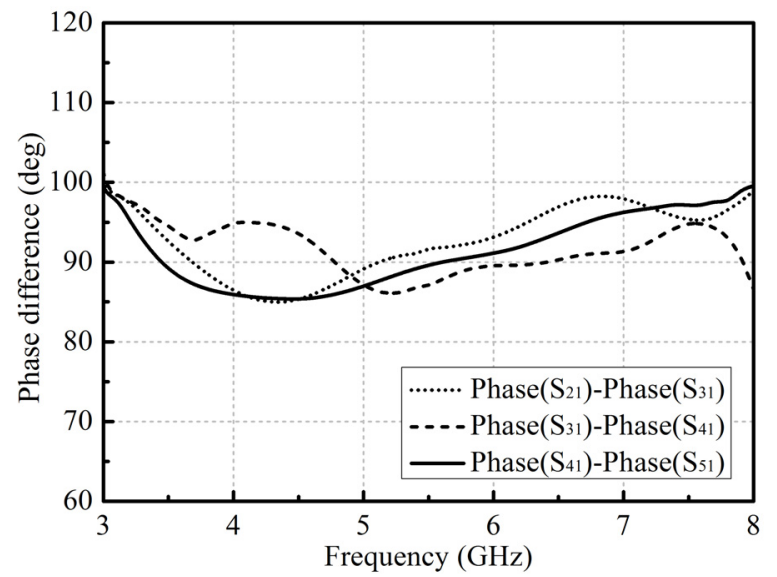

(b)

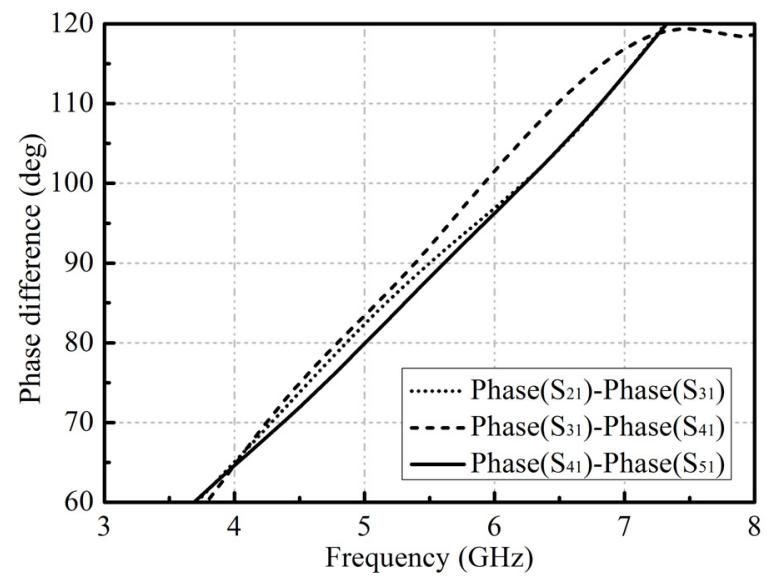

(d)

Fig. 7. Simulated results of SR feed network. (a) S-parameters for SR feed network based on the SCRLH-TLs. (b) Phase difference for SR feed network based on the SCRLH-TLs. (c) $S$-parameters for SR feed network based on the normal TLs. (d) Phase difference for SR feed network based on the normal TLs. 
ration. The easier the elements can be integrated for a network, the more simple the fabrication is, the less cost is.

From Fig. 7(a) and (b), it can be seen that the SR feed network using the SCRLH-TLs achieves a differential magnitude of $7.7 \pm 0.65 \mathrm{~dB}$ from $3.5 \mathrm{GHz}$ to $7.5 \mathrm{GHz}$ (the center frequency is $5.5 \mathrm{GHz}$ ) and a differential phase shift of $90^{\circ} \pm 10^{\circ}$ from $3 \mathrm{GHz}$ to $8 \mathrm{GHz}$ (the center frequency is also $5.5 \mathrm{GHz}$ ). To ensure the design antenna array with good performances, we chose $5.5 \mathrm{GHz}$ as center frequency for the antenna element and the antenna array. Based on the above design principles, a coupled stacked $\mathrm{CP}$ antenna element shown in Fig. 8 is designed in this paper. Because the coupled stacked structure can overcome several of the performance hindrances associated with direct contact excitation procedures (probe and edge feeding). These include the inherent narrow bandwidth of direct contact fed patches and also the spurious radiation associated with the current discontinuity where the feed and the patch join [14]. In our design, a square patch with a dimension of $L_{1} \times L_{1}$, which has a pair of symmetrically truncated patch corners, is etched between the bottom substrate and the middle substrate. The bottom substrate has a thickness of $h_{1}$ and a relative permittivity of $\varepsilon_{r 1}$, and the middle substrate is air with a thickness of $h_{2}$. The square patch is driven by a microstrip feed line. A parasitic patch element with a dimension of $L_{2} \times L_{2}$ is etched between the middle layer and the upper layer and a pair of patch corners is also symmetrically truncated on it. And the upper dielectric layer has a thickness of $h_{3}$ and a relative permittivity of $\varepsilon_{r 3}$. A branch on the feed line is used for impedance matching. The detail parameters are given as follows: $h_{1}=0.5 \mathrm{~mm}, \varepsilon_{r 1}=2.65$, $h_{2}=3.5 \mathrm{~mm}, \varepsilon_{r 2}=1, h_{3}=1.5 \mathrm{~mm}, \varepsilon_{r 3}=4.1, L_{1}=17.3 \mathrm{~mm}$, $L_{2}=17.2 \mathrm{~mm}, \Delta L_{1}=4 \mathrm{~mm}, \Delta L_{2}=17.3 \mathrm{~mm}, d_{1}=3.8 \mathrm{~mm}$, $l_{1}=3.25 \mathrm{~mm}, w_{1}=1.36 \mathrm{~mm}, w_{2}=1.36 \mathrm{~mm}$.

Figure 9 and 10 present the simulated VSWR and AR. The results indicate that the bandwidth of $\operatorname{VSWR}<2$ is essentially $25.45 \%$, from $4.8 \mathrm{GHz}$ to $6.2 \mathrm{GHz}$, and the bandwidth of $\mathrm{AR}<3 \mathrm{~dB}$ is $9.26 \%$, from $5.15 \mathrm{GHz}$ to $5.65 \mathrm{GHz}$. Figure 11 shows the gain of the stacked patch antenna element. It can be seen that the gain of the element varied between $7.75 \mathrm{dBic}$ and $10.1 \mathrm{dBic}$ across the frequency band between $4.5 \mathrm{GHz}$ to $6.5 \mathrm{GHz}$ and a peak gain of $10.1 \mathrm{dBic}$ appears at $6.1 \mathrm{GHz}$. The simulated results of the normalized electric field radiation patterns in two ortho-

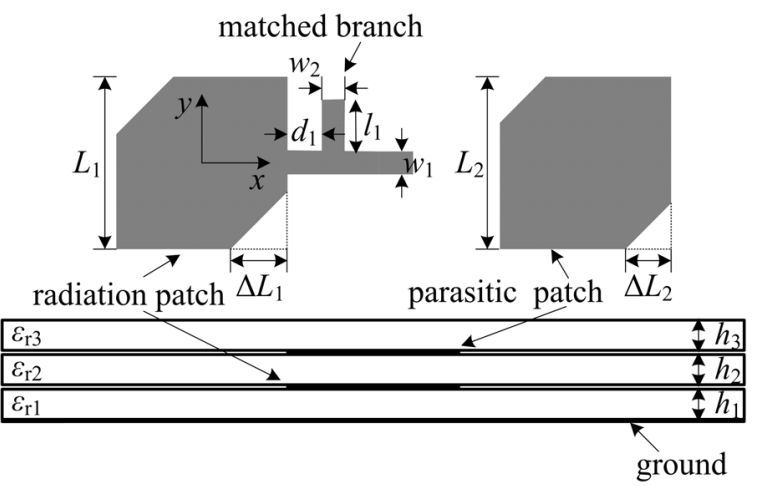

Fig. 8. Cross-sectional view of the stacked patch antenna element.

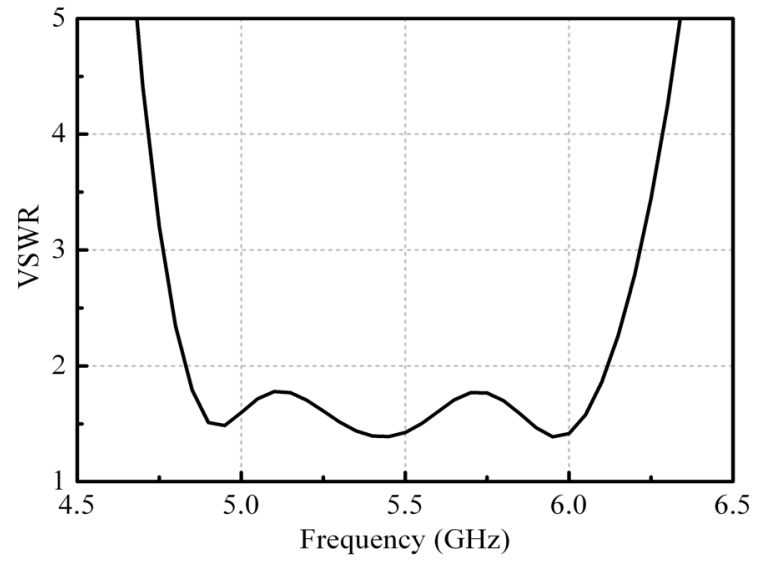

Fig. 9. Simulated VSWR for the antenna element.

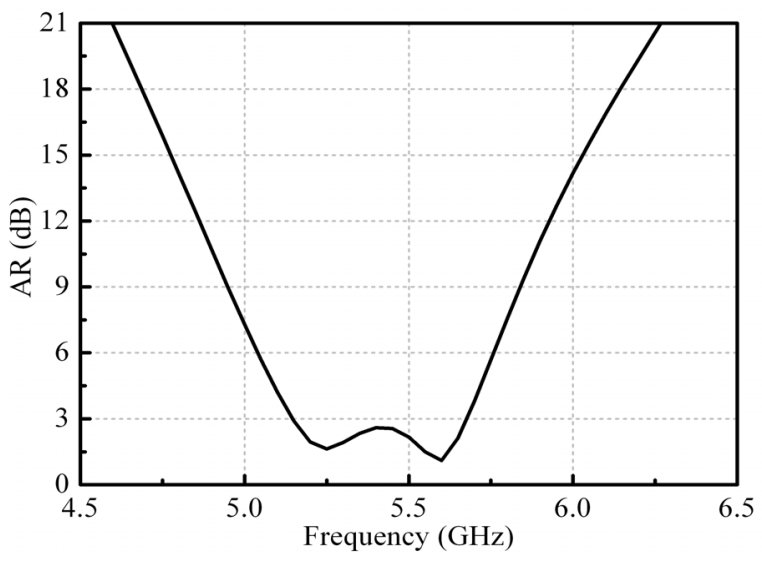

Fig. 10. Simulated AR for the antenna element.

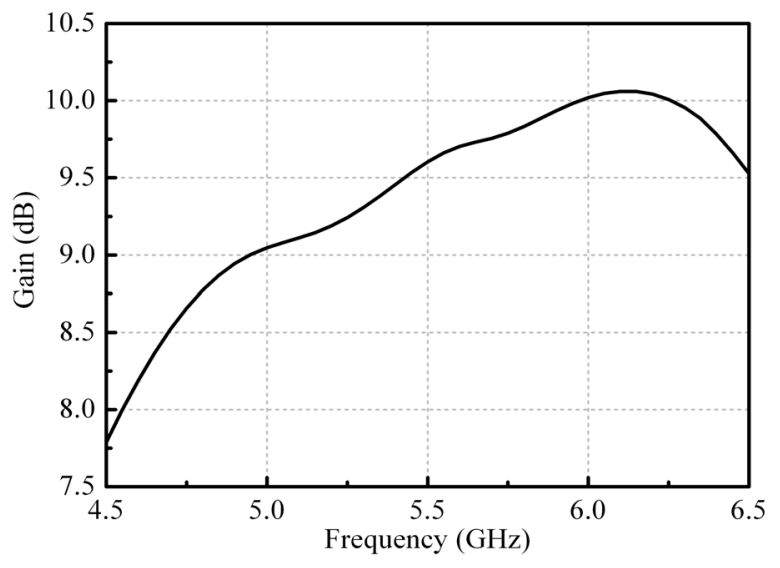

Fig. 11. Simulated gain for the antenna element.

gonal planes for the antenna at $5.15 \mathrm{GHz}, 5.5 \mathrm{GHz}$ and $5.65 \mathrm{GHz}$ are plotted in Fig. 12(a)-(c). It can be observed that good right-hand $\mathrm{CP}$ radiations are obtained and the cross-polarizations are low.

\section{Broadband CP Antenna Array}

Photographs of the fabricated $2 \times 2 \mathrm{CP}$ SR antenna array are shown in Fig. 13. The feed is attached to a connector through an opening in the ground plane. The VSWR of the proposed antenna array shown in Fig. 14 was meas- 


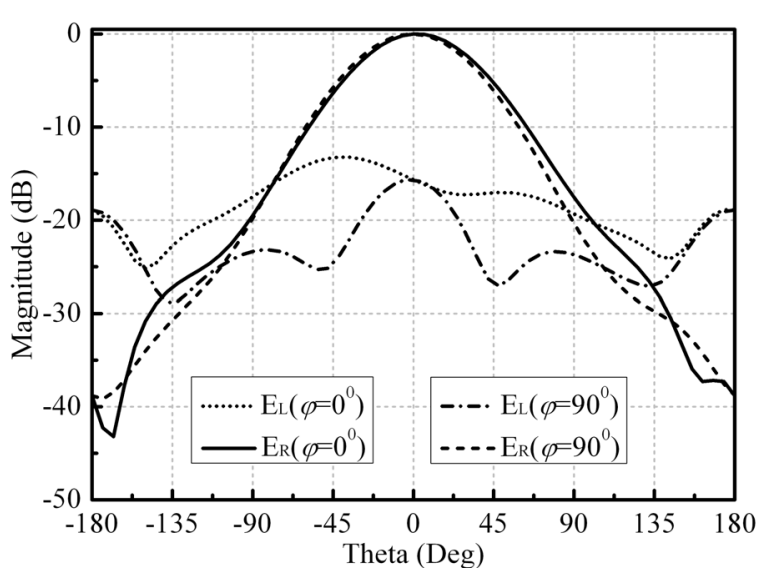

(a)

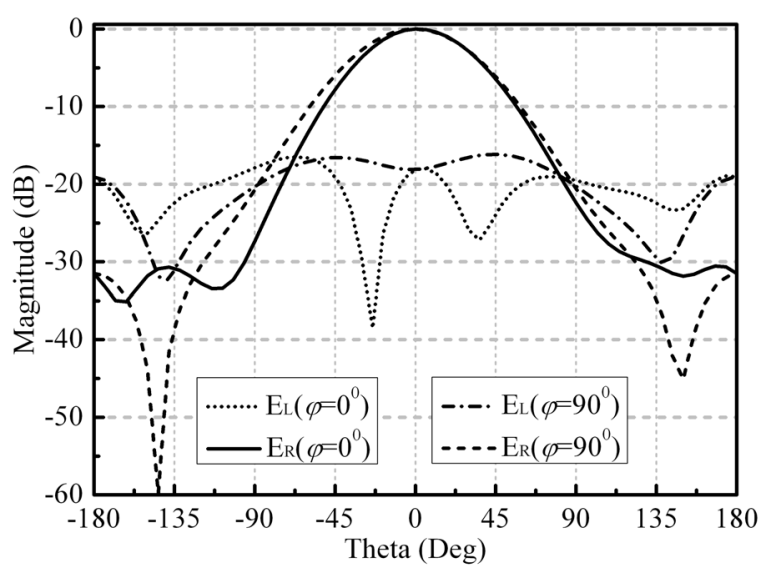

(b)

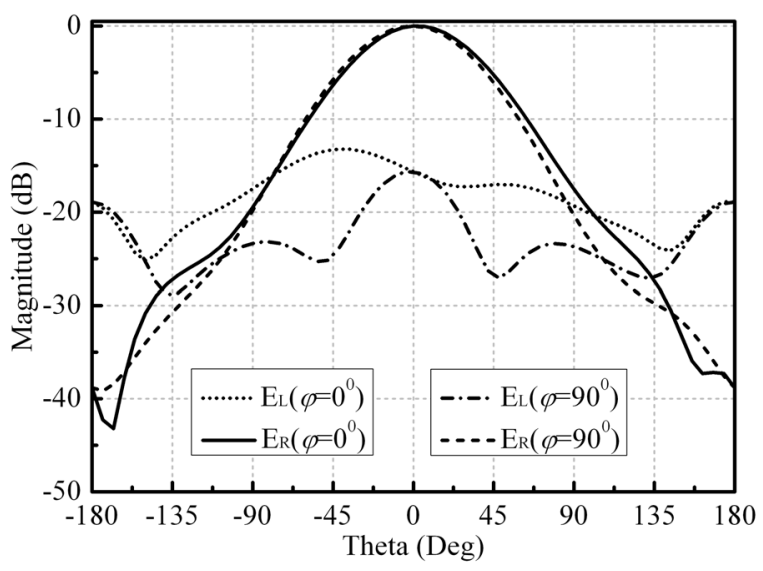

(c)

Fig. 12. Simulated normalized electric field radiation patterns of the antenna element. (a) $5.15 \mathrm{GHz}$, (b) $5.5 \mathrm{GHz}$, (c) $5.65 \mathrm{GHz}$.

ured using the Agilent ME7807M network analyzer. It can be seen that the measured VSWR is below 1.8 from 4 to $7 \mathrm{GHz}$. The VSWR is 1.39 at the center frequency $(5.5 \mathrm{GHz})$ and the minimum point of the curve appears at $4.5 \mathrm{GHz}$ with a magnitude of 1.09 . The simulated and measured results of the AR of the antenna array are depicted in Fig. 15. We can observe that the measured AR bandwidth of the array is $42.8 \%$ (from 4.4 to $6.8 \mathrm{GHz}$ ) with

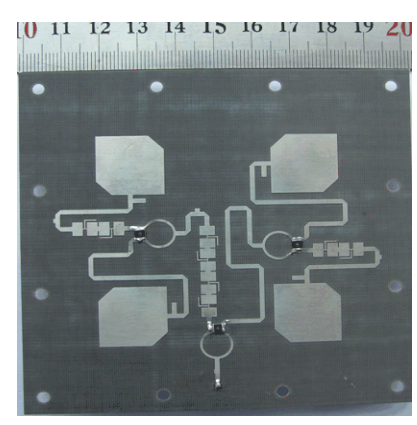

(a)

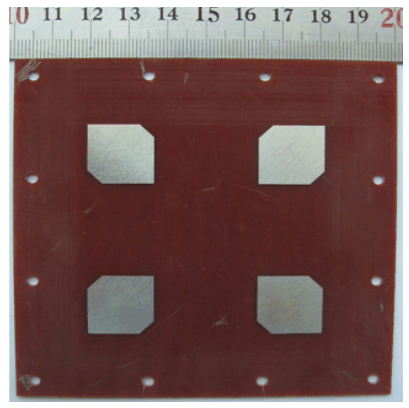

(b)

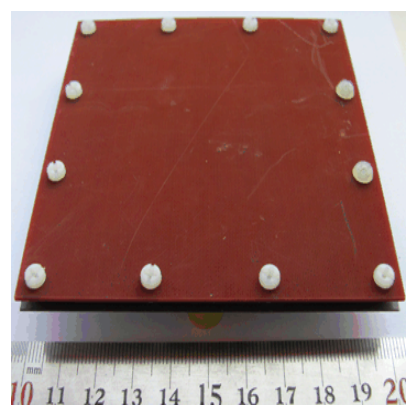

(c)

Fig. 13. Photographs of the fabricated $2 \times 2$ antenna array. (a) Exciting layer. (b) Parasitic layer. (c) Assembly.

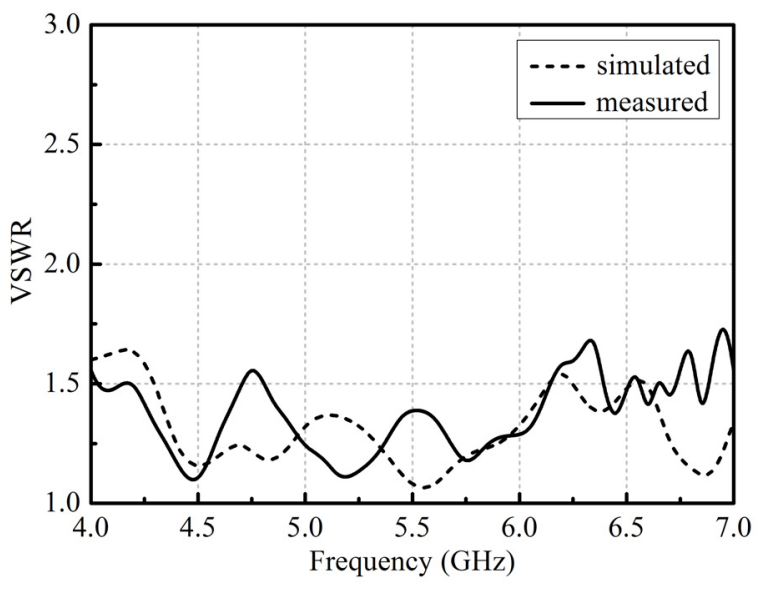

Fig. 14. Simulated and measured VSWR of the antenna array.

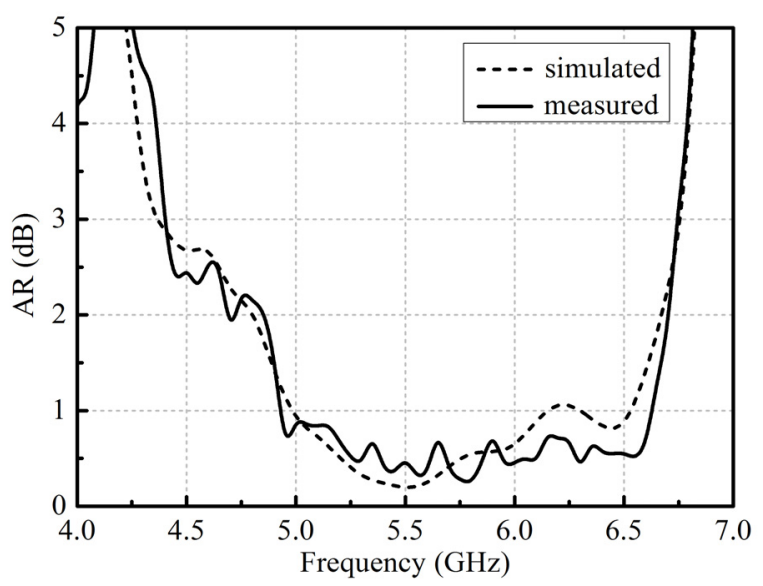

Fig. 15. Simulated and measured AR of the antenna array. 


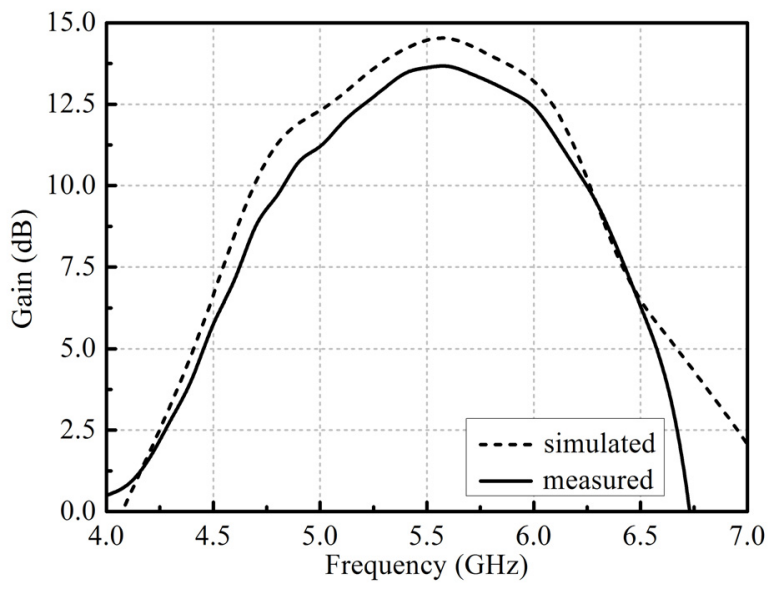

Fig. 16. Simulated and measured Gain of the antenna array.

$\mathrm{AR}<3 \mathrm{~dB}$ and is $32 \%$ (from 4.85 to $6.7 \mathrm{GHz}$ ) with $\mathrm{AR}<2 \mathrm{~dB}$. From 4.95 to $6.6 \mathrm{GHz}$, the measured $\mathrm{AR}$ is below $1 \mathrm{~dB}$. Compared with the antenna element, the antenna array has a wider 3-dB AR bandwidth, which indicates that the proposed SR feed network provides an excellent $\mathrm{CP}$ property in a wide bandwidth. Fig. 16 shows the simulated and measured gain of the proposed antenna array. A standard linearly polarized horn antenna was used to measure the total gain characteristics of the proposed design. The measured gain levels of the antenna array, which are generally lower than the simulated ones, varied between $5 \mathrm{dBic}$ and $13.7 \mathrm{dBic}$ from $4.46 \mathrm{GHz}$ to $6.58 \mathrm{GHz}$. The maximum difference between the simulated and measured gain is up to $1.1 \mathrm{dBic}$ at $5 \mathrm{GHz}$. The differences between the simulation and measurement could be attributed to fabrication imperfectness, material parameters inaccuracy, and measurement errors.

In Fig. 17, the simulated and measured results of the normalized radiation patterns of the array at $5.5 \mathrm{GHz}$ are presented. It can be seen that the normalized radiation patterns of the center frequency $5.5 \mathrm{GHz}$ are symmetrical at $\varphi=0^{\circ}$ and $\varphi=90^{\circ}$. The half-power beamwidths at $\varphi=0^{\circ}$ and $\varphi=90^{\circ}$ are all $28^{\circ}$. The sidelobe levels are $-16.33 \mathrm{~dB}$ and $-16.39 \mathrm{~dB}$ at $\varphi=0^{\circ}$ and $\varphi=90^{\circ}$, respectively. The normalized radiation patterns of the array at $5 \mathrm{GHz}$ and $6 \mathrm{GHz}$ have the same characteristics with those ones at $5.5 \mathrm{GHz}$. The antenna array has a total size of $100 \times 100 \mathrm{~mm}^{2}$. All of the results indicate that the proposed SR feed network has a good potential in designing a broadband $\mathrm{CP}$ antenna array.

\section{Conclusion}

A $2 \times 2 \mathrm{CP}$ antenna array comprising sequentially rotated coupled stacked CP antenna elements has been presented in this paper. The SR feed network is synthesized using a balun and two orthogonal power dividers. The balun is composed of a Wilkinson power divider and a broadband $90^{\circ}$ phase shifter, and the orthogonal power divider is composed of a Wilkinson power divider and a broadband $180^{\circ}$ phase shifter. The merits of the proposed antenna array include a relatively simple structure, low

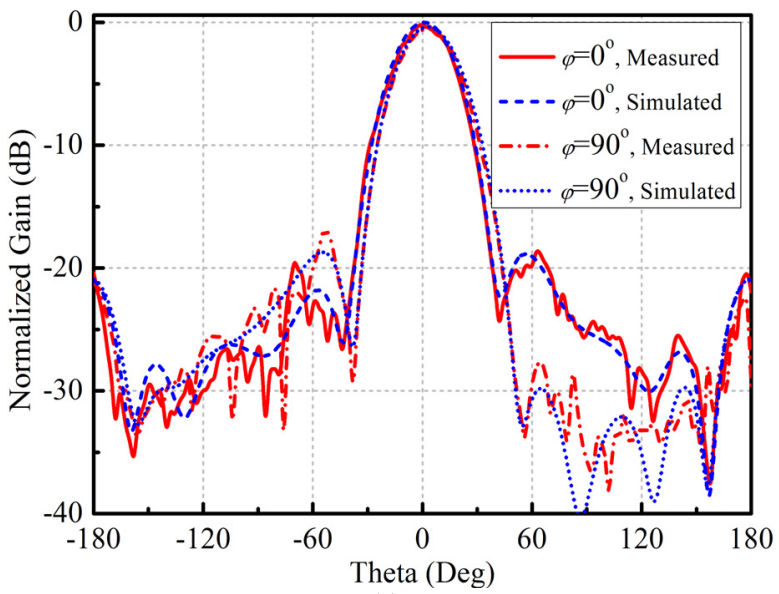

(a)

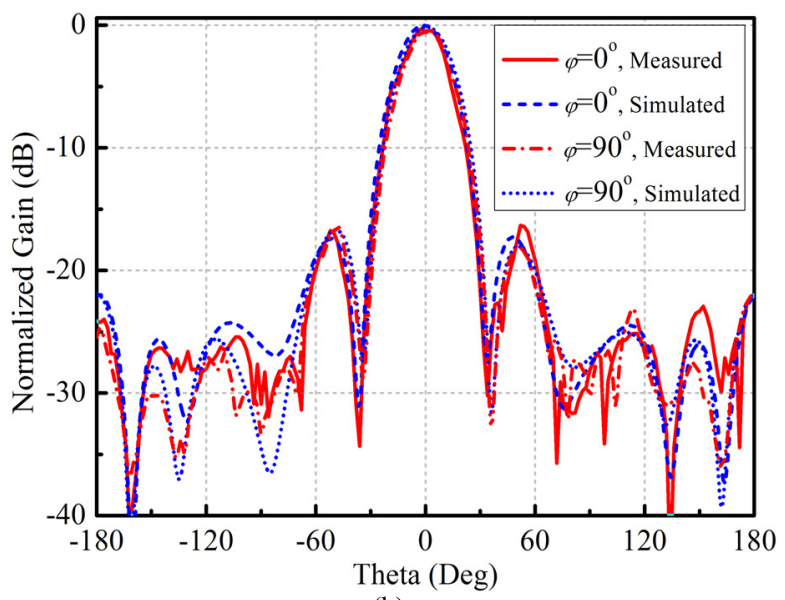

(b)

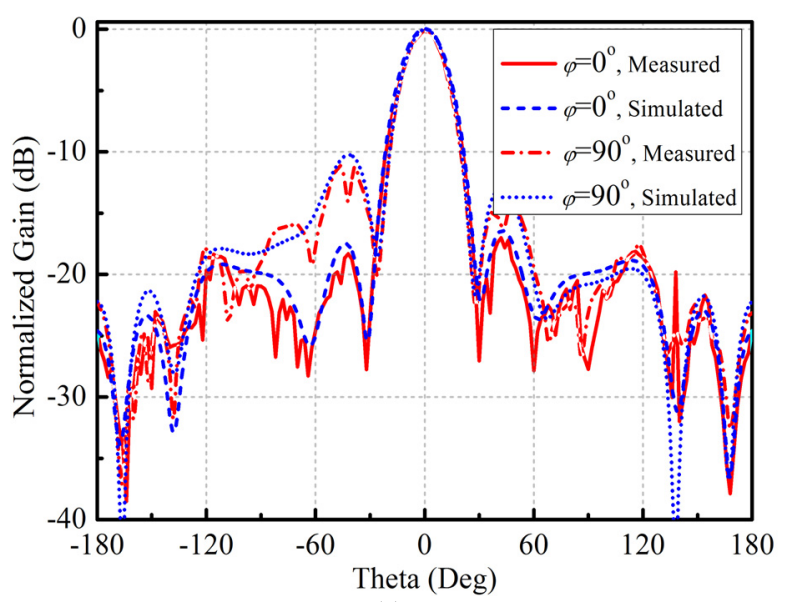

(c)

Fig. 17. Simulated and measured normalized radiation patterns of the antenna array. (a) $5 \mathrm{GHz}$. (b) $5.5 \mathrm{GHz}$. (c) $6 \mathrm{GHz}$.

fabrication cost, and broadband operation bandwidth. The measured results show that the bandwidths of the array with VSWR $<1.8$ is from 4 to $7 \mathrm{GHz}$ and with $\mathrm{AR}<3 \mathrm{~dB}$ is from 4.4 to $6.8 \mathrm{GHz}$. The maximum gain of the array is 13.7 dBic. From the comparison between the results of an antenna element and those of the antenna array, it can be known that the proposed SR feed network can enhance the VSWR and AR bandwidths significantly. 


\section{Acknowledgments}

This work is supported by the National Natural Science Foundation of China under Grant No. 61372034. The authors would also like to thank the China North Electronic Engineering Research Institute for the fabrication and measurement support.

\section{References}

[1] HUANG, J., POZAR, D.-M. Miscrotrip arrays: Analysis, design, and applications. In Advances in Microstrip and Printed Antennas, K. F. Lee and W. Chen, Eds. New York: Wiley-Interscience, 1997, p. 123.

[2] GUO, Y.-X., BIAN, L., SHI, X.-Q. Broadband circularly polarized annular-ring microstrip antenna. IEEE Transaction on Antennas and Propagation, 2009, vol. 57, no. 8, p. 2474-2477. DOI: 10.1109/TAP.2009.2024584

[3] HU, Y.-J., DING, W.-P., CAO, W.-Q. Broadband circularly polarized microstrip antenna array using sequentially rotated technique. IEEE Antennas and Wireless Propagation Letters, 2011, vol. 10, p. 1358-1361. DOI: 10.1109/LAWP.2011.2177799

[4] HALL, P.-S., HUANG, J., RAMMOS, E., ROEDERER, A. Gain of circularly polarized array composed of linearly polarized elements. Electronics Letters, 1989, vol. 25, no. 2, p. 124-125. DOI: $10.1049 / \mathrm{el}: 19890091$

[5] JAZI, M.-N., AZARMANESH, M.-N. Design and implementation of circularly polarized microstrip antenna array using a new serial feed sequentially rotated technique. IEE Proceedings - Microwave Antennas and Propagation, 2006, vol. 153, no. 2, p. 133-140. DOI: 10.1049/ip-map:20050005

[6] RAFII, V., NOURINIA, J., GHOBADI, C., POURAHMADAZAR, J., VIRDEE, B.-S. Broadband circularly polarized slot antenna array using sequentially rotated technique for C-band applications. IEEE Antennas and Wireless Propagation Letters, 2013, vol. 12, p. 128-131. DOI: 10.1109/LAWP.2013.2237744

[7] FU, S., FANG, S., WANG, Z., LI, X. Broadband circularly polarized slot antenna array fed by asymmetric CPW for L-band applications. IEEE Antennas and Wireless Propagation Letters, 2009, vol. 8, p. 1014-1016. DOI: 10.1109/LAWP.2009.2031662

[8] HUANG, J. A technique for an array to generate circular polarization with linearly polarized elements. IEEE Transaction on Antennas and Propagation, 1986, vol. 34, no. 9, p. 1113-1124. DOI: $10.1109 /$ TAP.1986.1143953

[9] CHEN, A.-X., ZHANG, Y.-J., CHEN, Z.-Z., CAO, S.-F. A Kaband high-gain circularly polarized microstrip antenna array. IEEE Antennas and Wireless Propagation Letters, 2010, vol. 9, p. 1115-1118. DOI: 10.1109/LAWP.2010.2093866

[10] YANG, S.-S., CHAIR, R., KISHK, A.-A., LEE, K.-F., LUK, K.M. Study on sequential feeding networks for subarrays of circularly polarized elliptical dielectric resonator antenna. IEEE Transaction on Antennas and Propagation, 2007, vol. 55, no. 2 , p. 321-333. DOI: 10.1109/TAP.2006.889819

[11] LIN, X.-Q., LIU, R.-P., YANG, X.-M., CHEN. J.-X., YIN, X.-X., CHENG, Q., CUI, T.-J. Arbitrarily dual-band components using simplified structures of conventional CRLH TLs. IEEE
Transactions on Microwave Theory and Techniques, 2006, vol. 54, no. 7, p. 2902-2909. DOI: 10.1109/TMTT.2006.877434

[12] GONG, J.-Q., CHU, Q.-X. SCRLH TL based UWB bandpass filter with widened upper stopband. Journal of Electromagnetic Waves and Applications, 2008, vol. 22, no. 14, p. 1985-1992. DOI: $10.1163 / 156939308787537982$

[13] POZAR, D.-M. Microwave Engineering. New York: Wiley, 2005.

[14] ROWE, W.-S.-T., WATERHOUSE, R.-B. Investigation into the performance of proximity coupled stacked patches. IEEE Transaction on Antennas and Propagation, 2006, vol. 54, no. 6, p. 1693-1698. DOI: 10.1109/TAP.2006.875462

\section{About the Authors ...}

Bin-Feng ZONG was born in Jiangsu province, People's Republic of China. He received his B.S. and M.S. degrees from the Air Force Engineering University, Xi'an, China, in 2010 and 2012, respectively. His research interests include microstrip resonators, composite right/left-handed (CRLH) transmission lines and metamaterial-based antennas.

Guang-Ming WANG was born in Anhui province, People's Republic of China. He received his B.S. and M.S. degrees from the Missile Institute of the Air Force Engineering University, Xi'an, China, in 1982 and 1990, respectively, and his Ph.D. degree from the Electronic Science and Technology University, Chengdu, China, in 1994. Then, he joined the Air Force Engineering University as a professor in 2000 and is now the head of the Microwave Laboratory center in it. His current interest includes microwave circuits, antenna and propagation, and also new structures including EBG, PBG, metamaterials and fractals, etc.

Hui-Yong ZENG was born in Henan province, People's Republic of China. He received his M.S. and Ph.D. degrees in 2010 and 2013, respectively. He is an engineer in Unit 93942. His research interests are metamaterials-based antennas, etc.

Ya-Wei WANG was born in Anhui province, People's Republic of China. He received his M.S. and Ph.D. degrees from the Air Force Engineering University, Xi'an, China, in 2011 and 2014, respectively. Now, he is a teacher of the Air Force Engineering University. His research interests are mainly spiral antennas, metamaterial-based antennas, etc.

Ding WANG was born in Shandong province, People's Republic of China. He received the M.S. degree in 2012. Now, he is a teacher of the Qingdao Campus of Naval Aero Engineering Acad. His research interests include aviation communication and navigation, and he is good at English. 